\title{
Computer Forensics and Image Deblurring: An Inclusive Investigation
}

\author{
Zohair Al-Ameen \\ ${ }^{1}$ Faculty of Computing, Universiti Teknologi Malaysia \\ ${ }^{2}$ Faculty of Information Sciences and Engineering, Management and Science University \\ E-mail: qizohair3@live.utm.my \\ Ghazali Bin Sulong \\ Faculty of Computing, Universiti Teknologi Malaysia \\ E-mail: ghazali@spaceutm.edu.my \\ Md. Gapar Md. Johar \\ Faculty of Information Sciences and Engineering, Management and Science University \\ Email: gapar@msu.edu.my
}

\begin{abstract}
Observed images with bare eyes are always different than the acquired ones using an imaging system since the captured images are considered as the degraded versions of the original scene. These degradations may vary between image noise, lighting defects and blur. Therefore, this article addresses the field of computer forensics with image deblurring as the latent details that are indeed present in the captured images are concealed due to the blurring artifact. Moreover, the constant types of blur that are being dealt with in forensics are the motion and the out-of-focus blur. The motion blur occurs due to the motion of the recorded objects or the camera during the capturing process. The out-of-focus blur occurs due to lens defocusing errors. Different examples are provided to focus on the importance of deblurring forensic images. In addition, concise commentaries on deblurring methods, applications and blur types are deliberated for additional knowledge.
\end{abstract}

Index Terms - Computer forensics; Image deblurring; Motion blur; Out-of-focus blur; Image degradations; Deblurring software.

\section{INTRODUCTION}

Cameras are used to record images that signify a correct representation of the original observed scene. However, every recorded image is more or less blurry. Thus, image deblurring is fundamental in making pictures sharp and useful. Image deblurring recovers the original, sharp image by using a mathematical model of the blurring process. The key issue is that some information on the lost details is indeed present in the blurred image but they are hidden and can only be recovered if known details of the blurring process are provided [1]. Generally, the recorded image can be expressed as [2]:

$G=H * F+N$
Where, $(\mathrm{F})$ is the original scene, $(\mathrm{H})$ is the point spread function (PSF), $(\mathrm{N})$ is the additive noise, $(*)$ is a convolution process and $(\mathrm{G})$ is the obtained degraded image [2]. Unfortunately, the original scene cannot be recovered exactly because of different unavoidable errors in the recorded image. Still, many challenges arise when trying to deblur images, such as devising efficient and reliable algorithms for recovering as much information as possible from the given data and a correct estimation of the blurring kernel. Many environments are used to develop the image processing applications; MATLAB is one of these applications that are widely used by different researchers. The basic MATLAB package contains many functions and tools for this purpose, but in some cases it is more convenient to use routines that are only available from the Signal Processing Toolbox (SPT) and the Image Processing Toolbox (IPT) [1]. Image deblurring signifies one of the most common cases of the applied inverse problems, with practical applications varying from medicine to forensics. Current studies in image deblurring are focused on iterative methods. Direct deblurring (Wienertype filtering in the Fourier domain) is limited by problems with spatially-invariant blur and stationary Gaussian noise [3]. Other methods such as blind deconvolution are also used to process various types of blurry images. The rest of the article takes the following order: in section two, the types of forensics blur are explained along with their causes. In section three, the deblurring methods used to reduce the blurring artifacts are briefly clarified. In section four, the famous forensics deblurring software are highlighted and brief descriptions about them are also provided. In section five, some forensic examples about degraded and deblurred images using different forensic software are also provided. Finally, in section six, a brief conclusion about this article is mentioned to highlight its important issues. 


\section{TYPES OF BLUR IN FORENSICS}

Usually, two types of blur are identified as the main source of image blurring that are motion and out-offocus blur. The motion blur happens due to many reasons such as the motion of objects during the image capturing procedure [4], the movement of cameras at the time of the scene capturing [5]. In addition, motion blur caused by a relative motion between a camera and a scene is inevitable due to the nature of a camera sensor that accumulates incoming light over a certain period of time [6]. Besides, the out-of-focus blur happens due to many reasons such as lens defocusing [7], and lens focusing error [8]. Due to the latter issues, the forensic images are degraded by blur. The complicated part is when an image has more than one type of blur, which occurs in most images. Current deblurring technology cannot solve the different blur types occurring in different parts of a single image, or on top of one another. For example, if an image of a moving car is captured and the camera was shaking during the image acquisition, the car will be blurry because of its motion and the whole image might have some blur due to the camera shake. If an image has other issues like the noise you often get from camera phones, or if it was taken in low light, the algorithms might identify the wrong parts of an image as blurry, and thus add artifacts in the deblur process that make it look worse.

\section{DEBLURRING METHODS}

In image processing, two types of methods are used to deblur images. These methods are called blind and nonblind deconvolution procedures [9]. The blind deconvolution is a technique that recovers the target scene from a single or a set of blurred images were, the point spread function (PSF) type is unknown. For blind deconvolution, the PSF is estimated from the image or image set, allowing the deconvolution to be performed [10]. Whereas, the non-blind deconvolution is the process of estimating the latent image from the blurred image with a known PSF such as motion PSF or Gaussian PSF [11]. Both blind and non-blind deconvolution methods can be performed iteratively, whereby each iteration improves the estimation of the PSF and the scene, or non-iteratively, where one application of the algorithm, based on exterior information, extracts the PSF.

\section{FORENSICS DEBLURRING APPLICATIONS}

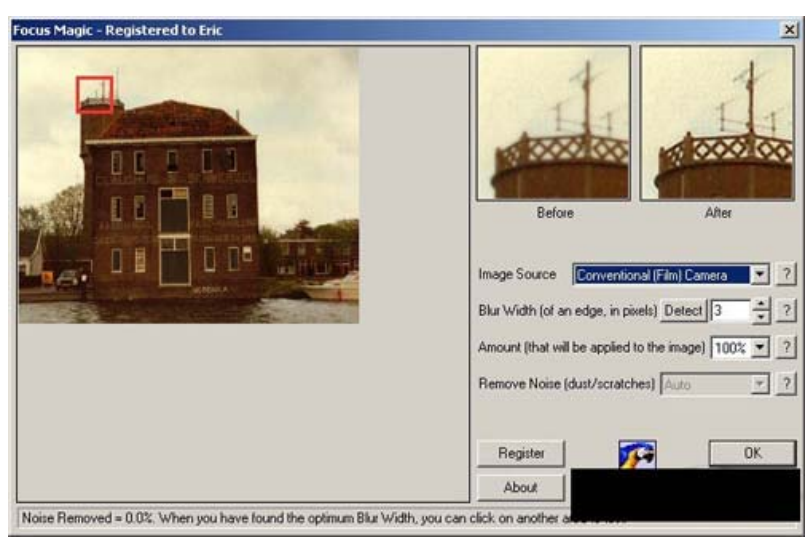

Figure 1. The Focus Magic Interface.

Different applications are used by the forensic specialists to deblur the captured images due to motion or out-of-focus blur. This article highlights a variety of common applications that are usually used in this field. The first software is Focus Magic. Focus Magic uses advanced forensic strength deconvolution technology to literally "undo" blur. It can repair both out-of-focus blur and motion blur (camera shake) in an image. It is the only software that can significantly recover lost detail from blurred images [12]. As shown in the Fig. 1, the interface is relatively simple and the outcome of the software is adequate. Finally, using this software only require some basic knowledge in the field of image deblurring to get the desired clear results.

The second software is ClearID Workflow which provides two methods for forensic deblurring, both based upon a technique called "Deconvolution." Basically this is a mathematical way to provide a corrective lens to the image to try to arrive at what the original image must have been like. This was used extensively on the pictures from the Hubble Telescope prior to the first service mission because the telescope was nearsighted. Smart Sharpen and ClearID's Interactive deblur approach deconvolution in different ways. Interactive deblur can yield better results for both motion blur and focus blur, but can also have a number of artifacts that are less than perfect. Smart Sharpen can yield some useful results with its "Lens Blur" setting, but these results are rarely as "sharp" as those from Interactive deblur. Both are provided in the hopes that ONE of them will work as shown in Fig. 2 [13]. 


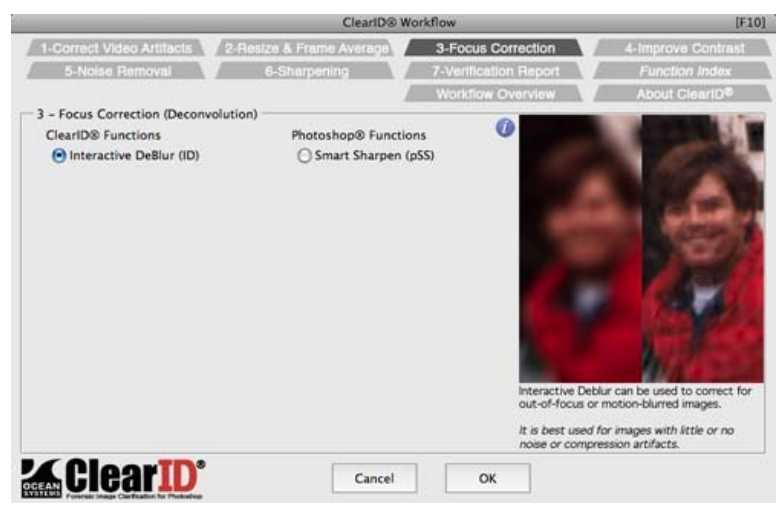

Figure 2. The ClearID Workflow Interface.

The third software is Amped Five which is a world leader in image processing software specifically designed for investigative, forensic and security applications. Its primary purpose is to provide forensic investigators a complete and unique solution to process and analyze digital images and video data in a simple, fast and precise way. Through processing and analyzing both still and video images, then utilizing a clear workflow to present evidence that withstands the scrutiny of the courtroom, Amped Five is the forensic investigator's trusted partner. Furthermore, Amped Five allows investigators to solve a wide range of problems which are otherwise easily missed. Since five is selfcontained, it can help to dramatically reduce the time required to process data and improves the success rate of the treated cases, from the restoration of low quality CCTV video to fingerprint analysis as displayed in Fig. 3 [14].

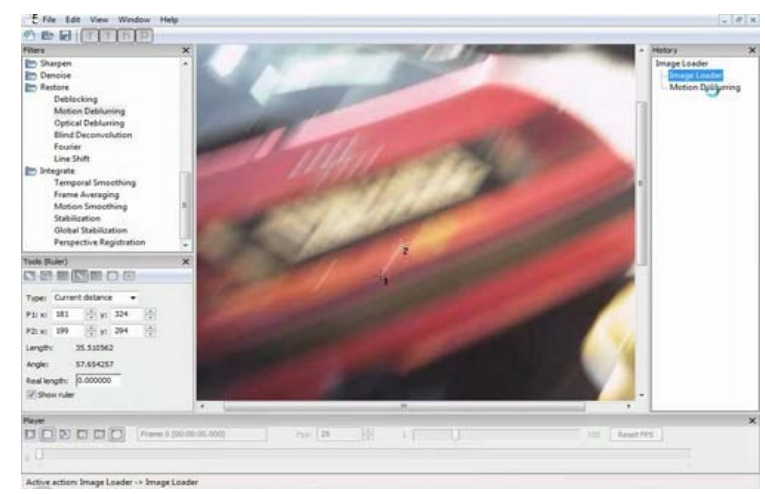

Figure 3. The Amped Five Interface.

The fourth software is Ikena Forensics. During the video capture process, a certain amount of blurring is introduced due to the low-pass nature of the optical system and video compressor. Ikena uses a special deblurring filter (bilateral total variation) that is able to sharpen images without the typical increase in noise seen with edge sharpening or unsharp mask. A standard unsharp mask is also available. Ikena can deinterlace interlaced video, such as DVD or security camera videos. Moreover, it uses super-resolution techniques to extract extra information from the two interlaced fields, treating each field as a separate frame, allowing Ikena to accurately fuse those fields into a single progressive image, without artifacts as presented in Fig. 4 [15].

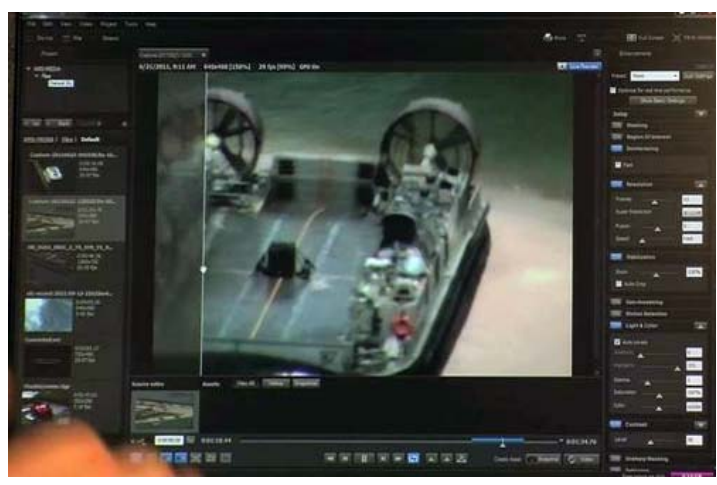

Figure 4. The Ikena Forensics Interface.

The fifth software is Cognitech Video Investigator. It is the latest major release of the world's first forensic video processing software suite, first released in mid1990. Cognitech Video Investigator Software is the state of the art video enhancement and processing software package. Incorporating over 40 plugins there is virtually nothing that cannot be achieved in regards to forensic video enhancements. Video Investigator will allow you to deblur, denoise, automatically track, reconstruct, mosaic, stabilize moving images, change intensity levels, brightness and colors, convert into any other common file format (avi, mpeg, tiff, jpeg, etc.), measure suspects, motion tracking, velocity computations, frame fusion and frame averaging, obscure and highlight faces and license plates, get component information, automated logging, scene descriptions and capture video scenes from propriety digital CCTV recorders in an uncompressed format as shown in Fig. 5 [16].

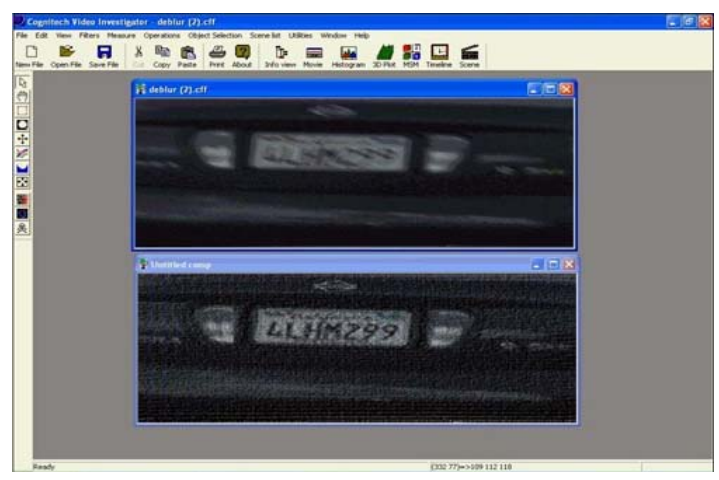

Figure 5. The Cognitech Investigator Interface.

The sixth software is Topaz InFocus where it uses a deconvolution technology to mathematically reverse image blur while increasing the actual sharpness. InFocus can enhance the clarity of an already wellfocused as well as deblur an out-of-focus or motion blurred image. The ability to re-focus a blurred image is a challenging but valuable capability. This technology can be applied to both enhancing the clarity in an already well-focused image, as well as de-blurring outof-focus or motion blurred images. A common side 
effect of the deconvolution process is deconvolution artifacts. These artifacts can appear as a collection of artifacts around the lines of details in an image. Topaz InFocus includes a parameter which designed to remove these artifacts as displayed in Fig. 6 [17].

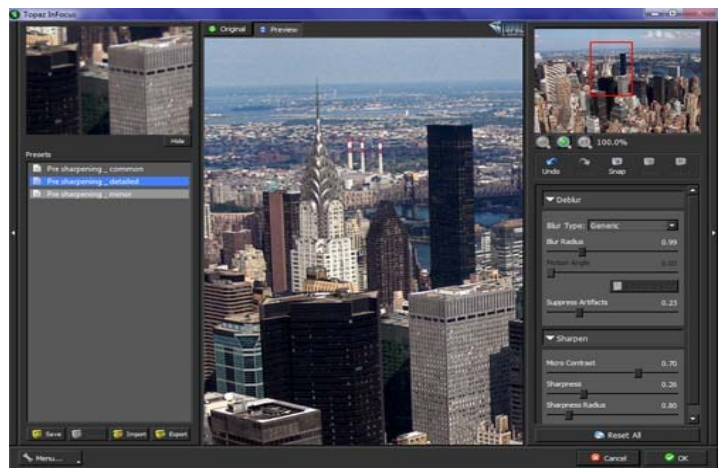

Figure 6. The Topaz InFocus Interface.

The seventh software is SmartDeblur where it uses a user friendly and useful tool for restoring blurry and defocused images. Blurry images are universal in our life. There are many causes of that: camera shakes, defocussing, and artificial blur via photo editors like Photoshop or Gimp. Many people think that blurring is an irreversible operation and the information in this case is lost for good. But it is not quite true. The new release features a blind deblurring module which can automatically identify and remove complicated blur patterns in an image as seen in Fig. 7 [18].

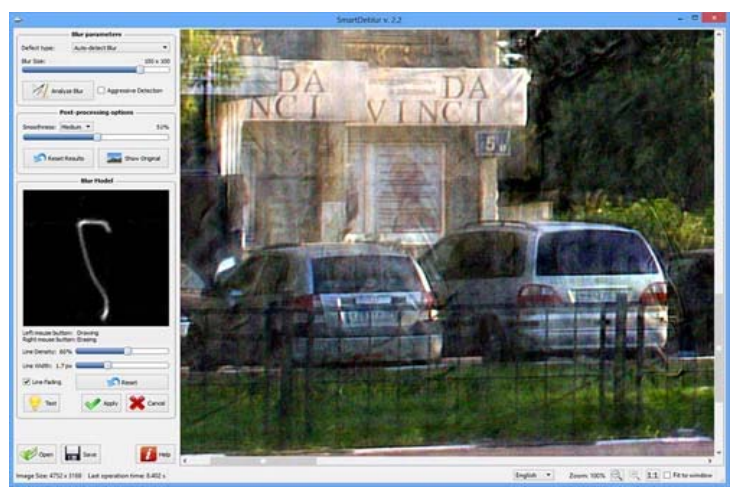

Figure 7. The SmartDeblur Interface.

The eighth software is Blurity where, this tool can remove the blur from any photo. All you need is a computer, a fuzzy photo, and the desire to see it sharp. Moreover, Blurity can be used with its default settings for an easy use. Also, a user can dive into the settings if the user is an expert in the deblurring field and wants to squeeze the best deblurring as displayed in Fig. 8 [19].

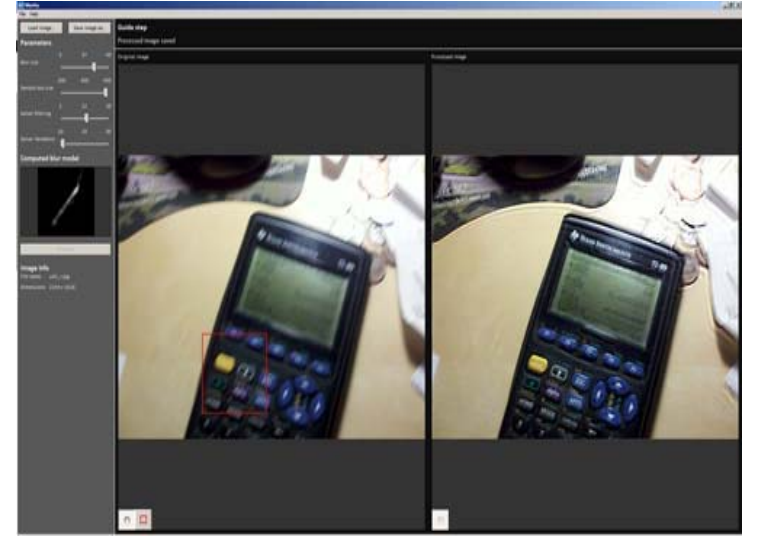

Figure 8. The Blurity Interface.

The ninth software is Back In Focus where this tool can quickly increase the sharpness of the processed photos by using the widely unsharp masking technique, which is a flexible and powerful way to increase sharpness. Furthermore, for more advanced refocusing, use one of the deconvolution algorithms to recover lost details. Back In Focus makes it easy to combine all refocusing techniques. Currently the implemented algorithms are: Unsharp masking (fast and full), Wiener finite and infinite impulse response, Richardson-Lucy (with a thresholding variant) and Linear algebra deconvolution as demonstrated in Fig. 9 [20].

The last software is FocusFixer where FixerLabs launched a Photoshop plug-in called FocusFixer. It has improved controls, is faster, has improved memory handling and claims to give better results than Unsharp Masking. The plug-in works with Photoshop $6 \& 7$ and is available for both Mac (OS X) and Windows. FocusFixer promises to fix blurred pictures. FixerLabs also claims to give clearer and sharper images than unsharp mask [21].

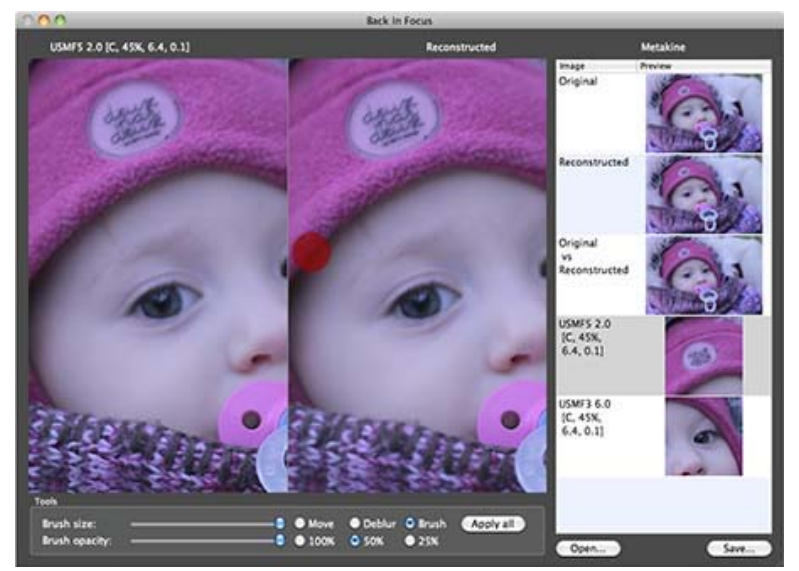

Figure 9. The Back In Focus Interface. 


\section{FORENSICS EXAMPLES}

In computer forensics, different software, algorithms and schemes are used to deblur the images that are degraded by different types of blur. The subsequent Figures are some examples that demonstrate certain images and their processed versions by different methods.
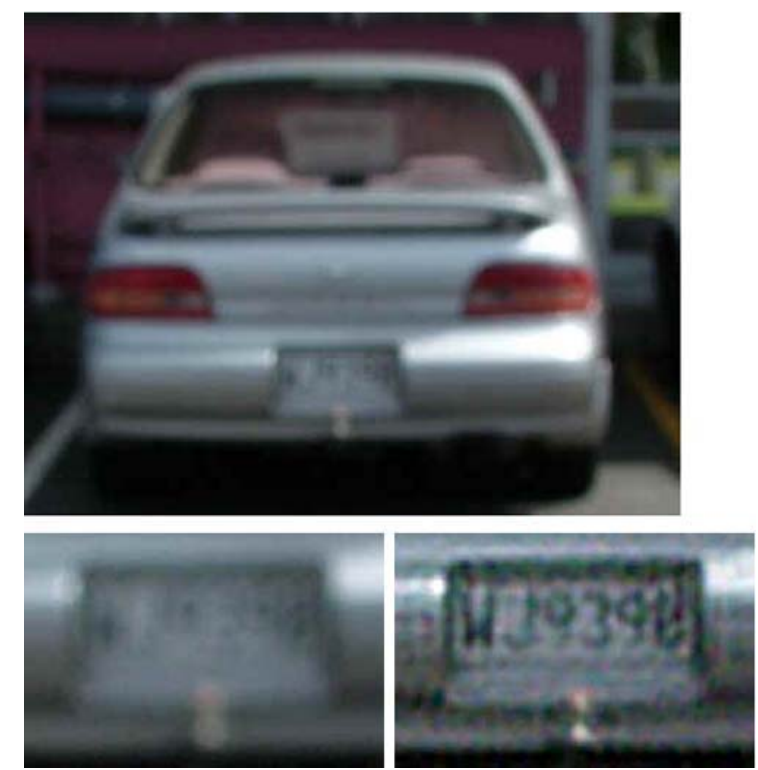

Figure 10. Images left to right: image degraded by out-of-focus blur; restored using Focus Magic [12].
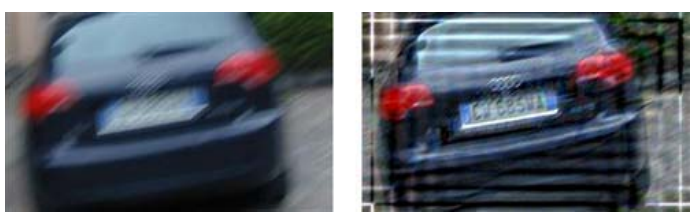

Figure 11. Images left to right: image degraded by motion blur; restored using Amped Five software [14].

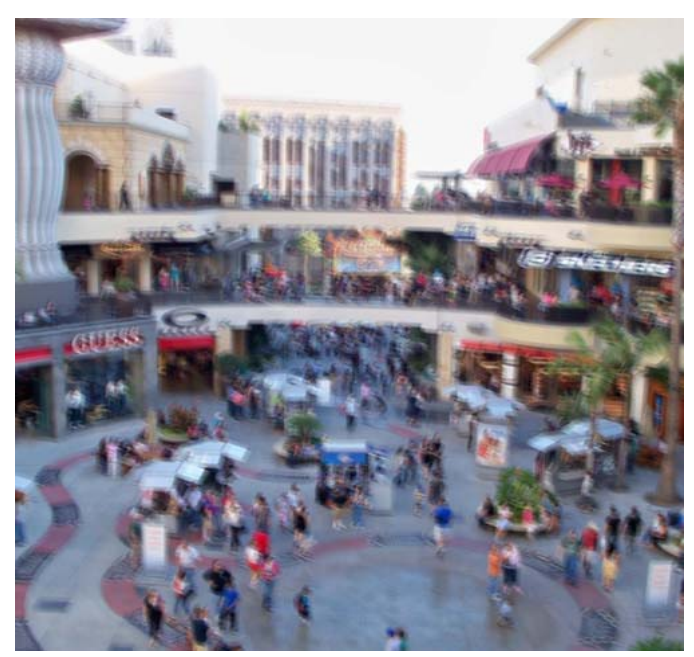

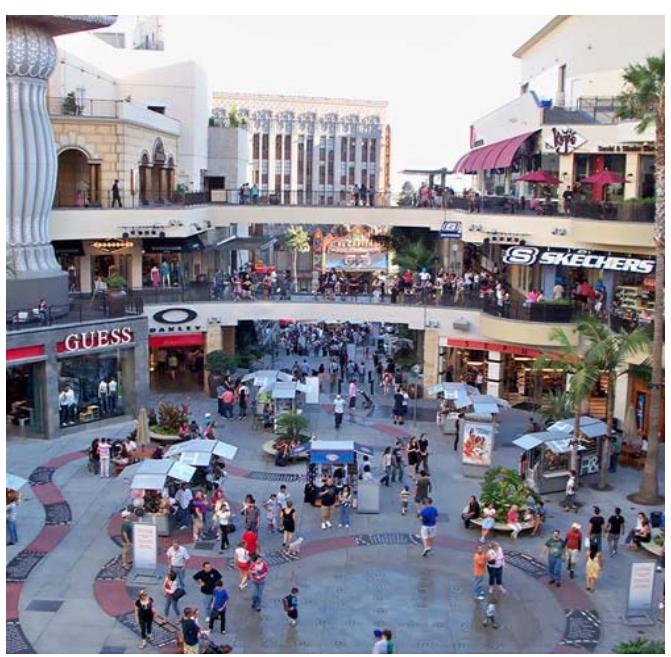

Figure 12. Images from top to bottom: image degraded by motion blur; restored using a specialized algorithm designed by Jue Wang [22].
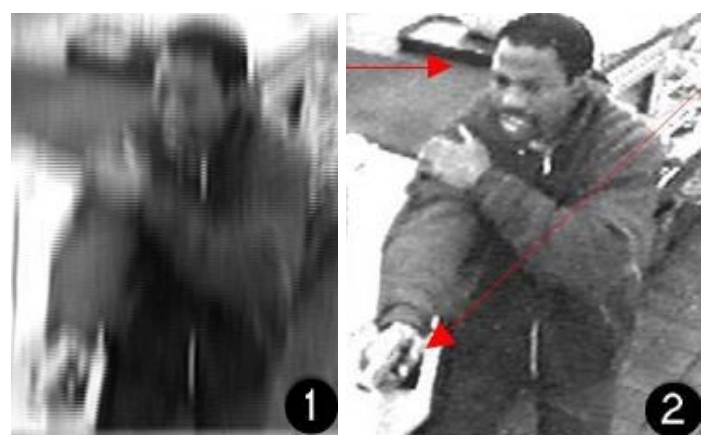

Figure 13. Images left to right: 1) a degraded still image of a typical CCTV camera; 2) restored using a specialized forensic algorithm [24].
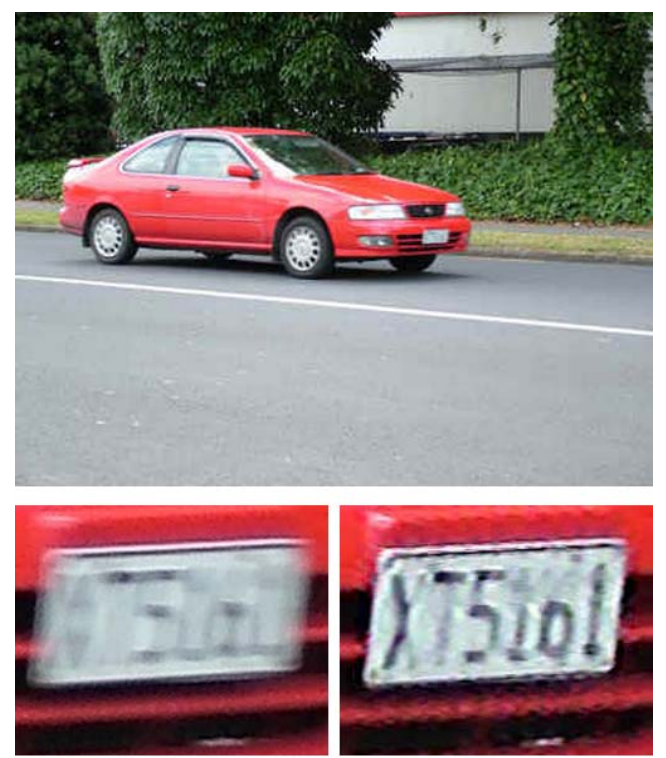

Figure 14. Images left to right: image degraded by motion blur; restored using Focus Magic [12]. 

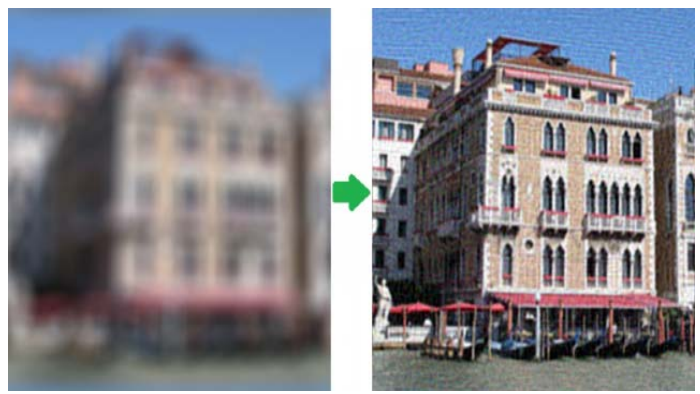

Figure 15. Images left to right: image degraded by out-of-focus blur; restored using SmartDeblur [25].
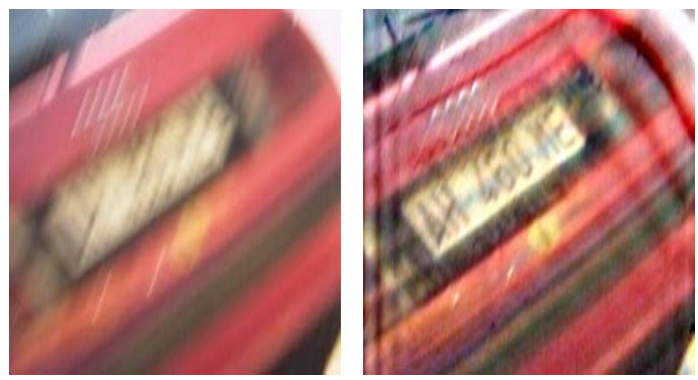

Figure 16. Images left to right: image degraded by motion blur; restored using Amped Five [14].

\section{CONCLUSION}

The leading purpose of this paper is to deliver a better understanding of deblurring images in the area of forensic science. As mentioned earlier, different types of blur may affect the recorded images. The types of blur that are usually dealt with in the forensic area are the motion and out-of-focus blur. Different labs, software companies and researchers are constantly aiming to develop new algorithms that are fast, reliable and accurate to improve the field of image deblurring. As a result, different deblurring applications were presented in this article to highlight that issue. Finally, these algorithms are not only used in forensics, but with a variety of imaging fields.

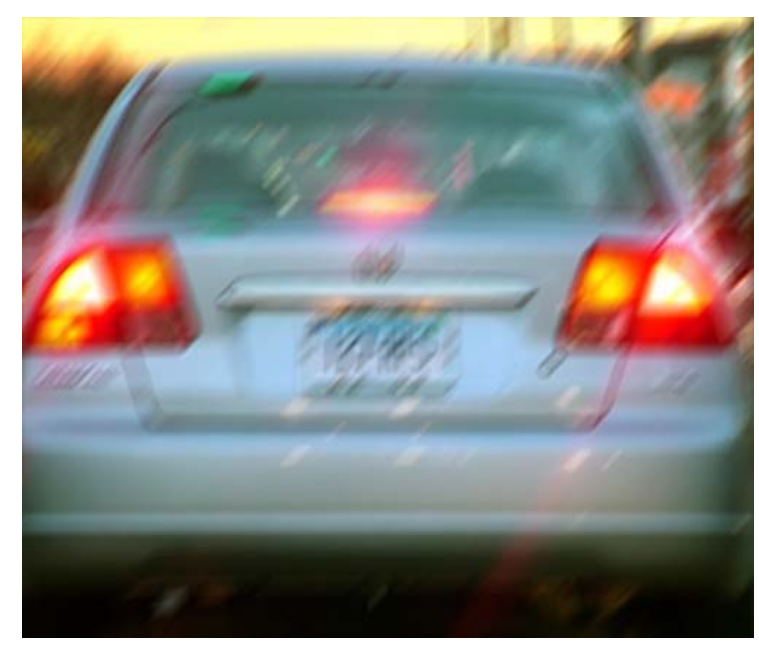

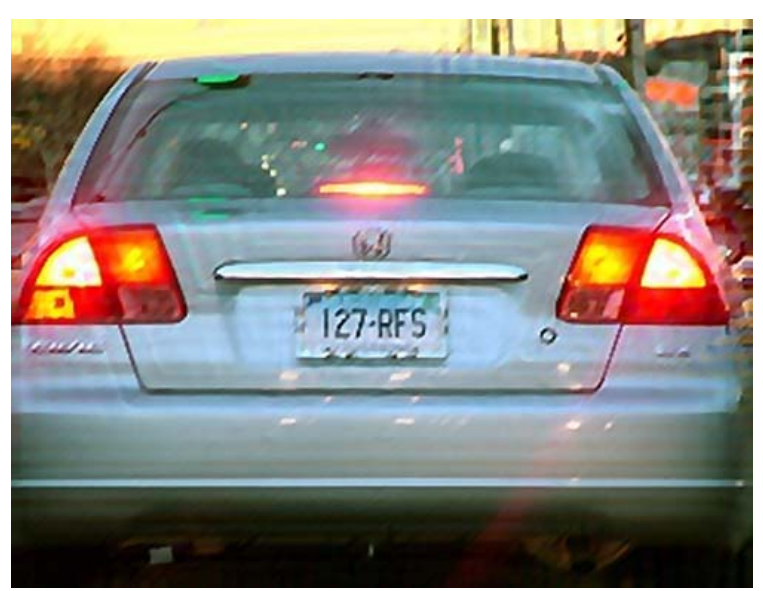

Figure 17. Images from top to bottom: image degraded by motion blur; restored using Topaz InFocus [23].
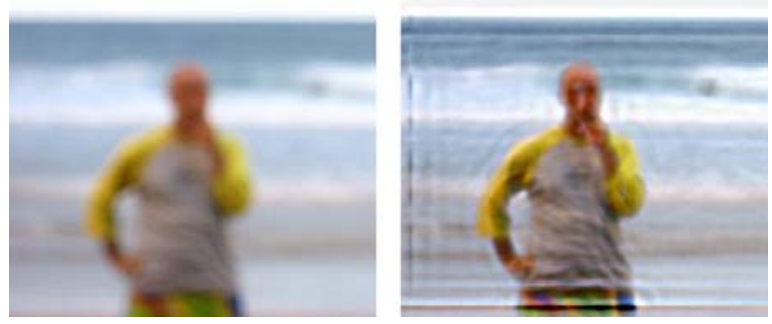

Figure 18. Images left to right: image degraded by out-of-focus blur; restored using Amped Five [14].

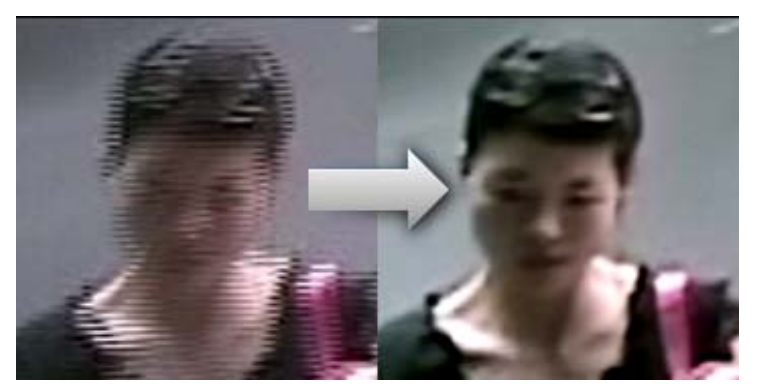

Figure 19. Images left to right: image degraded by motion blur; deblurred and deinterlaced using Ikena Forensics [15].

\section{REFERENCES}

[1] P. C. Hansen, J. G. Nagy and D. P. O'Leary, " Deblurring Images: Matrices, Spectra, and Filtering ", Society for Industrial and Applied Mathematics (SIAM), Philadelphia, USA, 1, 2006.

[2] Z. Al-Ameen, D. Mohamad, M. Shafry M. R. and G. Sulong, "Restoring Degraded Astronomy Images using a Combination of Denoising and Deblurring Techniques”, International Journal of Signal Processing, Image Processing and Pattern Recognition, 5(1), 2012.

[3] V. N. Strakhov and S. V. Vorontsov, "Digital image deblurring with SOR”, Inverse Problems, 24(2), 2008. 
[4] M. Potmesil and I. Chakravarty. "Modeling motion blur in computer-generated images", ACM SIGGRAPH Computer Graphics, 17(3), 389-399, 1983.

[5] R. Raskar, A. Agrawal and J. Tumblin, "Coded exposure photography: motion deblurring using fluttered shutter." In ACM Transactions on Graphics, 25(3), 795-804, 2006.

[6] S. Cho, Y. Matsushita and S Lee, "Removing nonuniform motion blur from images."IEEE 11th International Conference on Computer Vision, 1-8. 2007.

[7] M. E. Moghaddam, "Out of Focus Blur Estimation Using Genetic Algorithm”, Journal of Computer Science, 4(4), 298-304, 2008.

[8] S. H. Lim, J. Yen and P. Wu, "Detection of out-offocus digital photographs." Hewlett-Packard Laboratories Technical Report HPL, 14, 2005.

[9] M. Wilson, "Non-Blind and Blind Image Deconvolution.” CGU Math 336 Image Processing, 2011.

[10]E. Lam and J. W. Goodman,"Iterative statistical approach to blind image deconvolution".Journal of the Optical Society of America A, 17(7), 1177-1184, 2000.

[11] S. Cho, J. Wang and S. Lee, "Handling outliers in non-blind image deconvolution." IEEE International Conference on Computer Vision, 495502. 2011.

[12]Focus Magic Website: "http://www.focusmagic.com".

[13]ClearID Workflow Website: "http://reindeergraphics.com/forensics.html".

[14]Amped Five Website: "http://ampedsoftware.com/five".

[15]Ikena Forensics Website: "http://www.motiondsp.com/products/ikena/deblurand-deinterlace".

[16] Cognitech Video Investigator Website: “cognitechvideo-investigator.software.informer.com/”.

[17] Topaz InFocus Website: "http://www.topazlabs.com/infocus/".

[18] SmartDeblur Website: “http://smartdeblur.net/”.

[19] Blurity Website: "https://www.blurity.com/features”.

[20]Back In Focus Website: "http://www.metakine.com/products/backinfocus/".

[21]Focus Fixer Website: "http://www.photoi.co.uk/Reviews /software/Focusfixer/FocusFixernew.htm”.

[22] Jue Wang Adobe Webpage: "http://www.adobe.com/technology/people/seattle/j ue-wang.html”.

[23] Topaz Labs official Website: "www.topazlabs.com/ home.php”.

[24]Spencer Research Limited Website: "www.spencerresearch.co.uk/ videodeblurring.html”.

[25] Vladimir Yuzhikov, SmartDeblur 2.1 Website: "yuzhikov.com/index.html".
Zohair Al-Ameen was born in the United Kingdom in 1985, obtained his B.Sc. degree in computer science from the University of Mosul - IRAQ in 2008. In 2011 he obtained his M.Sc. in computer science from Universiti Teknologi Malaysia. His research interests include image and video processing, image restoration, image enhancement, medical image processing, segmentation, optical characters recognition, motion detection, and pattern recognition. Currently he is a full time Ph.D. student at the faculty of computing, Universiti Teknologi Malaysia.

Prof. Dr. Ghazali Bin Sulong received his BSc degree in statistics from National University of Malaysia, in 1979, and MSc and PhD in computing from University of Wales, Cardiff, United Kingdom, in 1982 and 1989, respectively. He is currently professor at faculty of computing, Universiti Teknologi Malaysia. His research interest includes biometric - fingerprint identification, face recognition, iris verification, ear recognition, handwritten recognition, and writer identification; object recognition; image enhancement and restoration; medical imaging; human activities recognition; data hiding - digital watermarking, steganography and image encryption; image fusion; image mining; digital image forensics; object detection, segmentation and tracking.

Prof. Dr. Md. Gapar Md. Johar A certified ecommerce consultant, he has over 30 years working experience in software and application development. His research interests include object-oriented analysis and design, software engineering, Java programming, digital image processing, Radio Frequency Identification (RFID) and knowledge management. Currently, he is the vice president academic of the Management and Science University (MSU). He holds BSc in computer science, $\mathrm{MSc}$ in data engineering and $\mathrm{PhD}$ in computer science. 